\title{
Variabilidad del viento, oleaje y corrientes en la región norte de los fiordos Patagónicos de Chile
}

\author{
Variability of wind, waves and currents in the northern region of the Chilean Patagonian fjords \\ Jaime Letelier ${ }^{1,2}$, Luis Soto-Mardones ${ }^{3}$, Sergio Salinas ${ }^{1}$, Pedro Osuna
Diego López
}

${ }^{1}$ Escuela de Ciencias del Mar, Pontificia Universidad Católica de Valparaíso, Av. Altamirano 1480, Valparaíso, Chile. jaime.letelier@ucv.cl, jaime.letelier@gmail.com

${ }^{2}$ Facultad de Ciencias del Mar y de Recursos Naturales, Universidad de Valparaíso, Casilla 5080 Reñaca, Viña del Mar, Chile ${ }^{3}$ Departamento de Física, Facultad de Ciencias, Universidad del Bío-Bío, Av. Collao 1202, Casilla 5-C, Concepción, Chile ${ }^{4}$ Departamento de Oceanografía Física, CICESE, Carretera Ensenada-Tijuana 3918, Zona Playitas 22860, Ensenada, B.C., México

${ }^{5}$ Departamento de Geofísica, Universidad de Concepción, Avda. Esteban Iturra s/n, Barrio Universitario, Concepción, Chile ${ }^{6}$ Instituto de Fomento Pesquero, Centro de Acuicultura Putemún, Putemún, Chile

${ }^{7}$ Instituto Antártico Chileno, Plaza Muñoz Gamero 1055, Punta Arenas, Chile

\begin{abstract}
Measurements of currents, waves and winds were made in Seno de Reloncaví, Corcovado Gulf, (Pacific Coast/west area) of Chiloé and the Darwin Canal inlet. These observations were complemented with historical data, with the aim of determining wind, current and wave extreme values and local variability north of the Patagonic fjords in Chile. Results showed the predominance of the north wind and local wave regime $(\mathrm{Hs}<0.5 \mathrm{~m})$ for the north coast of Seno de Reloncaví. The west of Chiloé is characterized by long waves (Hs $>2 \mathrm{~m}$ ) originating in the Pacific and the dominance of the south and southeast wind driven by regional circulation. In the area of Corcovado Gulf and the Darwin Canal inlet, the wind follows the direction of the topography, while at Italia Island local waves are predominant $(\mathrm{Hs}<0.5 \mathrm{~m})$. In Corcovado Gulf a combined local and long wave regime $(\mathrm{Hs} \sim 2 \mathrm{~m})$ was observed. Furthermore, results showed that the tide is the main coastal current forcing mechanism in the area of the fjords and canals, differing from that at Chiloé and Italia Island (Pacific Coast/west area), where wind is the main driver of coastal circulation. Differences found between both zones, especially between inlet and off the coast zones, north of the Patagonic fjords in Chile, are evidence that the topography plays a fundamental role in the wind and current directions.
\end{abstract}

Key words: Chiloé, currents, waves

Resumen.- Se realizaron mediciones de corrientes, oleaje y viento en el seno de Reloncaví, el golfo Corcovado, la zona exterior de Chiloé y en isla Italia, al interior del canal Darwin. Estas observaciones fueron complementadas con registros históricos con el objetivo de determinar los valores extremos y la variabilidad local del viento, las corrientes y el oleaje de la zona del norte de los fiordos patagónicos de Chile. Los resultados mostraron el predominio del viento norte y oleaje local $(\mathrm{Hs}<0,5 \mathrm{~m})$ en la costa norte del seno de Reloncaví. El sector occidental de Chiloé se caracteriza por oleaje largo $(\mathrm{Hs}>2 \mathrm{~m})$ proveniente del Pacífico y el dominio del viento sur-suroeste que es controlado por la circulación regional. En la zona del golfo Corcovado y al interior del Canal Darwin el viento y las corrientes siguen la dirección de la topografía. En isla Italia dominó el oleaje local ( $\mathrm{Hs}<0,5 \mathrm{~m}$ ) y en el golfo Corcovado se observó una combinación de oleaje local y oleaje largo (Hs $\sim 2 \mathrm{~m}$ ). Por otra parte, los resultados mostraron que la marea es el principal forzante de las corrientes costeras dentro del golfo Corcovado a diferencia de la zona exterior de Chiloé e isla Italia donde el viento es el principal conductor de la circulación costera. Las diferencias encontradas entre zonas y especialmente entre el interior y exterior de la zona del norte de los fiordos patagónicos de Chile mostraron que la topografía de la zona juega un rol fundamental en orientación del viento y las corrientes.

Palabras clave: Chiloé, corriente, olas 


\section{INTRODUCCIÓN}

En la zona interior y exterior de la región norte de fiordos patagónicos de Chile se han concentrado los estudios oceanográficos principalmente en el fiordo de Reloncaví (Valle-Levinson et al. 2007), mientras que en otras zonas la investigación científica ha estado dispersa en tiempo y espacio asociada mayoritariamente a los proyectos CIMAR-FIORDOS (Silva \& Palma 2006a). La extensión y la complejidad topográfica de la zona (Rodrigo 2006) y la diversidad de mecanismos oceanográficos-atmosféricos que se generan en esta zona hace que los experimentos realizados y la información disponible sea insuficiente para responder al interés científico y económico de la región (Silva \& Palma 2006b).

Recientes investigaciones han mostrado (ValleLevinson et al. 2007, González \& Cáceres 2009, SotoMardones et al. 2009, Cáceres et al. 2010, Castro et al. 2011) que la zona protegida del seno de Reloncaví actúa como sistema de circulación semi-expuesto con un importante aporte del fiordo de Reloncaví y una conexión con el golfo de Ancud a través de pasos estrechos (Nao, Queullín y Tautil). En esta zona, el viento y el oleaje es determinado por características topográficas locales y las conexiones con el estuario y el golfo (Soto-Mardones et al. 2009). Las intensas corrientes de marea $\left(>4 \mathrm{~m} \mathrm{~s}^{-1}\right)$ que ingresan por el estrecho canal de Chacao (Cáceres et al. 2003) al golfo de Ancud se bifurcan hacia el seno de Reloncaví con intensidades sobre los $0,4 \mathrm{~m} \mathrm{~s}^{-1}$ (SotoMardones et al. 2009) y hacia el sur por el canal Desertores con magnitudes sobre $0,7 \mathrm{~m} \mathrm{~s}^{-1}$ (Salinas \& Castillo 2011) 1 . La literatura disponible no reporta más información de corrientes hacia las zonas más expuestas (Golfo Corcovado y región exterior de Chiloé).

Por otro lado, las zonas adyacentes al seno de Reloncaví, como el golfo de Ancud, el golfo Corcovado y la región exterior de Chiloé carecen de información integrada que permita caracterizar de mejor manera los patrones de viento, de circulación y del oleaje. Solamente con aproximaciones analíticas, utilizando modelos simples, se han realizado estimaciones para tratar determinar el desarrollo del oleaje (Hasselmann et al. 1973, Massel 1996). De similar manera, operacionalmente se utiliza la semejanza con otras zonas para extrapolar patrones de circulación a falta de información, como en la zona interior del golfo Corcovado la que estaría dominada por la influencia directa del viento y el oleaje proveniente del Océano Pacífico a través de la boca o entrada del Guafo, similar a lo que ocurre con la boca del Golfo de
California en el Pacifico norte (Castro et al. 1994, Beier 1997, Beier \& Ripa 1998, Soto-Mardones et al. 1999, Berón-Vera \& Ripa 2000, Castro 2001, Berón-Vera \& Ripa 2002) Además, la marea debería seguir siendo un factor determinante en la circulación (Cáceres et al. 2007, Aiken 2008)

Evidencias de la intensa influencia del sistema oceánico regional sobre la costa del continente sudamericano se han observado y modelado en la zona exterior de Chiloé (Bakun \& Nelson 1991, Strub et al. 1998, Leth 2000). El régimen extremo de oleaje que se ha registrado instrumentalmente ha sido con altura que puede superar los $7 \mathrm{~m}$ en el sector oeste de la entrada del canal Chacao (Cabezas 1991), mientras que en Chile central alcanza $\sim 4 \mathrm{~m}$ (Belmar 2002). La escasa información oceanográfica y meteorológica de la zona ha obligado a usar normas noruegas como referencia para el desarrollo de ingeniería y de las normas ambientales chilenas. Dada las características particulares que diferencian la zona de fiordos de Chile de la zona de fiordos de Noruega (latitud, orientación de la costa, batimetría, topografía, masas de agua y circulación regional del viento y corrientes) se plantea como objetivo de este trabajo determinar mediante mediciones directas los valores extremos y la variabilidad local del viento, las corrientes y el oleaje de la zona del norte de los fiordos patagónicos de Chile.

\section{Materiales y Métodos}

Las zonas estudiadas comprenden parte norte del seno Reloncaví, costa oceánica noroeste de la isla de Chiloé, las costas oeste y este del golfo Corcovado y la zona interior del canal Darwin (Fig. 1). El enfoque del análisis realizado involucra información histórica y registros adquiridos en 5 campañas de mediciones. Datos históricos de oleaje en golfo Coronado provienen del Centro Nacional de Datos Hidrográficos y Oceanográficos (CENDHOC), mientras que datos históricos de vientos en Puerto Montt, isla Laytec y faro Corona provienen del Servicio Meteorológico de la Armada (SERVIMET). El resto de las estaciones (puntas Guabún, Chiguao, Auchemó e isla Italia al interior del canal Darwin) corresponden a campañas oceanográficas (corrientes, olas y viento) de más de 20 días de mediciones (Tabla 1). Además, se incluyen solamente 7 días de mediciones de olas y 30 días de viento en punta Metri debido a fallas en la instrumentación.

${ }^{1}$ Salinas S \& M Castillo. 2011. Corrientes mareales y submareales en el Canal Desertores ( $\left.42^{\circ} 42^{\prime} S, 72^{\circ} 50^{\prime} \mathrm{W}\right)$. Ciencia y Tecnología del Mar. [Aceptado] 
Tabla 1. Ubicación geográfica de las estaciones, variables registradas, periodo de muestreo y altura promedio de la columna de agua $(\mathrm{H})$ / Location of stations, variables, time period of measurements and water column height $(\mathrm{H})$

\begin{tabular}{|c|c|c|c|c|c|c|c|c|}
\hline Estación & Variable & $\begin{array}{l}\text { Longitud } \\
\text { (O) }\end{array}$ & $\begin{array}{l}\text { Latitud } \\
\qquad(\mathrm{S})\end{array}$ & $\begin{array}{c}\text { Inicio } \\
\text { (ddmmaa) }\end{array}$ & $\begin{array}{l}\text { Término } \\
\text { (ddmmaa) }\end{array}$ & $\begin{array}{l}\text { Período } \\
\text { (días) }\end{array}$ & $\mathrm{H}(\mathrm{m})$ & Topografía \\
\hline Punta Metri & $\begin{array}{c}\text { Olas } \\
\text { Viento }\end{array}$ & $\begin{array}{l}72^{\circ} 42^{\prime} 42,7^{\prime \prime} \\
72^{\circ} 42^{\prime} 28^{\prime}\end{array}$ & $\begin{array}{l}41^{\circ} 35^{\prime} 20,3^{\prime}, \\
41^{\circ} 35^{\prime} 20,9^{\prime},\end{array}$ & $\begin{array}{l}25062009 \\
25062009\end{array}$ & $\begin{array}{l}02072009 \\
25072009\end{array}$ & $\begin{array}{r}7 \\
30\end{array}$ & 16,2 & $\begin{array}{l}\text { Expuesto al Seno por } \\
\text { el sur. Plataforma } \\
\text { estrecha con pendiente } \\
\text { abrupta }\end{array}$ \\
\hline $\begin{array}{l}\text { Puerto } \\
\text { Montt* }\end{array}$ & Viento & 7257’39' & $41^{\circ} 29^{\prime} 5^{\prime \prime}$ & 01012007 & 30092008 & 638 & & $\begin{array}{l}\text { Expuesto al Seno por } \\
\text { el sur. Plataforma con } \\
\text { pendiente suave }\end{array}$ \\
\hline $\begin{array}{l}\text { Punta } \\
\text { Chiguao }\end{array}$ & $\begin{array}{l}\text { Corriente, } \\
\text { Olas, Viento }\end{array}$ & $73^{\circ} 27^{\prime} 59,2^{\prime \prime}$ & $43^{\circ} 8^{\prime} 46,5^{\prime \prime}$ & 13082009 & 04092009 & 21 & 11,7 & $\begin{array}{l}\text { Expuesto al golfo por } \\
\text { el este. Plataforma } \\
\text { amplia y pendiente } \\
\text { suave }\end{array}$ \\
\hline $\begin{array}{l}\text { Isla } \\
\text { Laytec* }\end{array}$ & Viento & $73^{\circ} 36^{\prime} 0^{\prime \prime}$ & $43^{\circ} 20^{\prime} 0^{\prime \prime}$ & 10082004 & 25112004 & 107 & & $\begin{array}{l}\text { Expuesto al golfo por } \\
\text { el este. Plataforma } \\
\text { amplia y pendiente } \\
\text { suave }\end{array}$ \\
\hline Auchemó & $\begin{array}{l}\text { Corriente, } \\
\text { Olas, Viento }\end{array}$ & $72^{\circ} 50^{\prime} 40,8^{\prime \prime}$ & $43^{\circ} 1^{\prime} 27,3^{\prime \prime}$ & 24112010 & 06012011 & 43 & 21,1 & $\begin{array}{l}\text { Expuesto al golfo por } \\
\text { el oeste. Plataforma } \\
\text { estrecha y pendiente } \\
\text { suave }\end{array}$ \\
\hline $\begin{array}{l}\text { Punta } \\
\text { Guabún }\end{array}$ & $\begin{array}{l}\text { Corriente, } \\
\text { Olas, Viento }\end{array}$ & $74^{\circ} 3^{\prime} 15,77^{\prime \prime}$ & $41^{\circ} 47^{\prime} 56,54^{\prime \prime}$ & 10092009 & 02102009 & 22 & 21,3 & $\begin{array}{l}\text { Expuesto al Pacífico. } \\
\text { Plataforma estrecha y } \\
\text { pendiente abrupta }\end{array}$ \\
\hline $\begin{array}{l}\text { Faro } \\
\text { Corona* }\end{array}$ & Viento & $73^{\circ} 53^{\prime} 18^{\prime \prime}$ & $41^{\circ} 47^{\prime} 6^{\prime \prime}$ & 01012007 & 31082008 & 608 & & $\begin{array}{l}\text { Abierto al océano. } \\
\text { Plataforma amplia y } \\
\text { pendiente suave }\end{array}$ \\
\hline $\begin{array}{l}\text { Golfo } \\
\text { Coronado* }\end{array}$ & Olas & $73^{\circ} 56,5^{\prime}$ & $41^{\circ} 40^{\prime}$ & 13051986 & 11061987 & 395 & 70 & $\begin{array}{l}\text { Abierto al océano. } \\
\text { Plataforma amplia y } \\
\text { pendiente suave. } \\
\text { Entrada canal Chacao }\end{array}$ \\
\hline Isla Italia & $\begin{array}{l}\text { Corriente, } \\
\text { Olas, Viento }\end{array}$ & $74^{\circ} 9^{\prime} 21,6^{\prime \prime}$ & $45^{\circ} 22^{\prime} 27,84^{\prime \prime}$ & 17102009 & 08022010 & 289 & 17,8 & $\begin{array}{l}\text { Interior de Canal con } \\
\text { plataforma estrecha y } \\
\text { pendiente abrupta }\end{array}$ \\
\hline
\end{tabular}

Las mediciones directas de corrientes y olas se realizaron con un correntómetro acústico ADCP RDI de $600 \mathrm{kHz}$, el cual fue puesto dentro de un soporte metálico para ser anclado al fondo del mar con el transductor mirando hacia arriba a $0,8 \mathrm{~m}$ desde el fondo. Este instrumento se configuró para efectuar 60 perfiles de corrientes con una resolución vertical de $0,5 \mathrm{~m}$ para luego promediar y registrar las muestras cada $10 \mathrm{~min}$, y por otra parte, efectuó cada $2 \mathrm{~h}$ y durante $20 \mathrm{~min}, 2400$ muestras de parámetros de olas. El sensor de presión incorporado en el ADCP permitió efectuar registros de la altura de la columna de agua con la misma resolución temporal de las mediciones de corrientes. Luego este parámetro es utilizado como criterio para seleccionar las capas de aguas que nunca fueron afectadas por las mareas. Finalmente, la columna de agua usada para los análisis de corrientes fue la confinada entre la zona de blanqueo y la penúltima capa observada. Además de eliminar la capa más superficial, de acuerdo con el programa computacional del ADCP, se eliminaron las observaciones de corrientes que presentaron un error mayor a $8 \mathrm{~cm} \mathrm{~s}^{-1}$ con este tipo de procedimientos se intenta eliminar los posibles datos contaminados debido a la interacción de la señal acústica con burbujas de aire proveniente del oleaje u otro (Teledyne RD Instruments) ${ }^{2}$.

${ }^{2}$ Teledyne Technologies Incorporated, Poway <http: / / www.rdinstruments.com> 


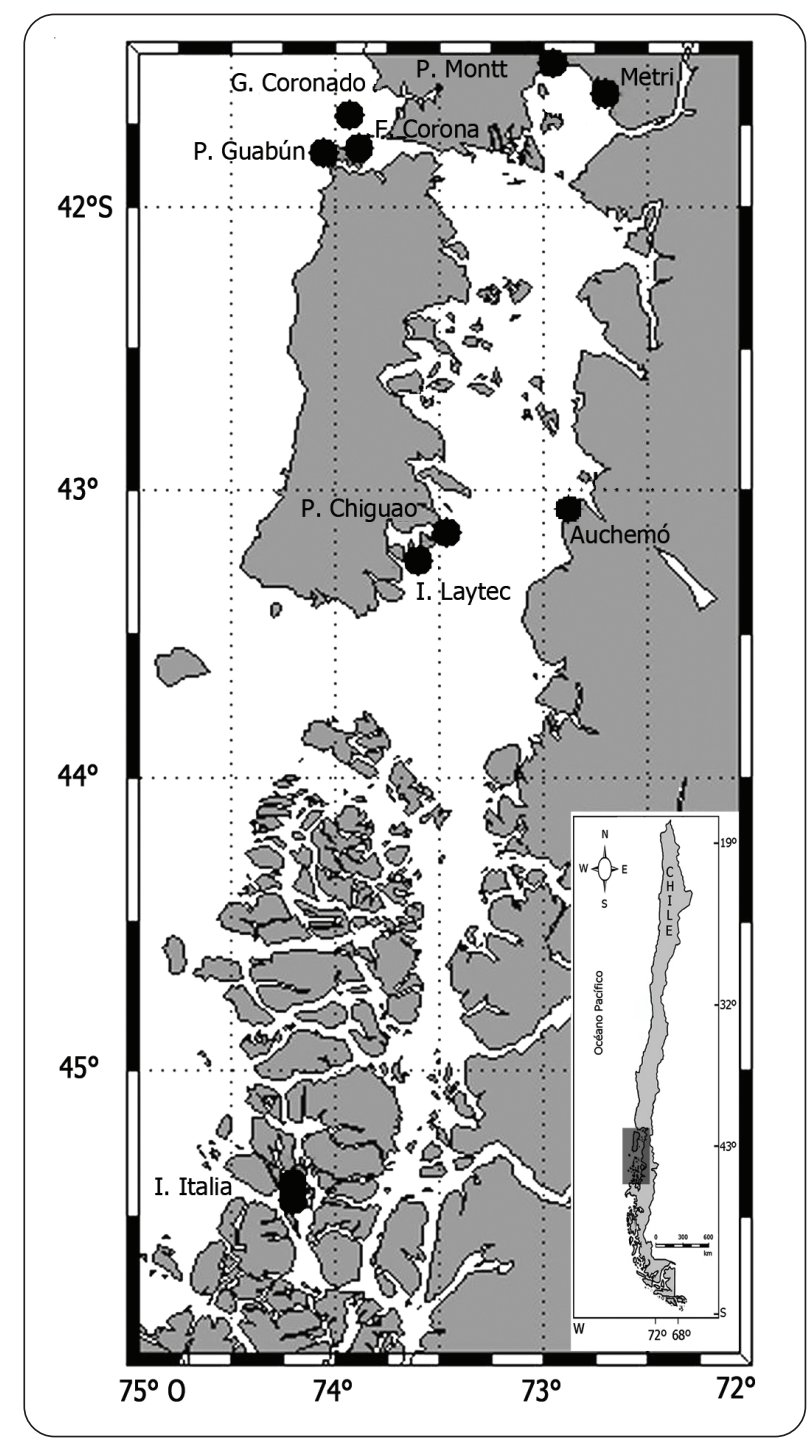

Figura 1. Ubicación geográfica de las estaciones en el mar interior de Chiloé, sur de Chile / Geographic location of the stations in the inland sea of Chiloé, southern Chile

Adyacente al lugar de mediciones costeras, se registró el viento utilizando un anemómetro Campbell Scientific Datalogger CR200, el cual se posicionó a $\sim 10$ metros sobre el nivel medio del mar. El anemómetro fue configurado para medir la rapidez y dirección del viento cada $10 \mathrm{~s}$ para luego ser promediadas y registradas en intervalos de $5 \mathrm{o}$ $10 \mathrm{~min}$. Las magnitudes de viento inferiores o iguales a 1 $\mathrm{m} \mathrm{s}^{-1}$ fueron considerados calmas.

La dirección de vientos y corrientes fue corregida con la desviación magnética de las respectivas zonas de estudio. En un análisis preliminar de las componentes de las corrientes se observó que dependiendo de la estación la mayor parte de la variabilidad no seguía las coordenadas norte-sur o este-oeste sino que la orientación de la costa, por lo que ambas componentes fueron rotadas siguiendo la elipse de los eje de máxima y mínima variabilidad. Esto se realizó descomponiendo las series originales en componentes ortogonales en las cuales se determina el ángulo de máxima y mínima variabilidad en todas las profundidades (Pizarro et al. 1994, Salinas \& Hormazábal 2003). Con los registros de corrientes se realizó un análisis armónico utilizando como entrada el algoritmo de Pawlowicz (2002) el vector complejo de la forma $w=u+v i$. El algoritmo utilizado para estimar la amplitud y fase se basa en el algoritmo descrito por Godin (1972), Foreman (1977) y Foreman (1978) implementado por Pawlowicz (2002). Por lo tanto se definió la corriente total $\left(\mathrm{V}_{\mathrm{T}}\right)$ asociada a la corriente directa medida por el ADCP, es decir, la corriente por marea más la componente residual $\left(\mathrm{v}_{\mathrm{r}}\right)$ asociada a otros mecanismos (viento, topografía, inercial, etc).

El análisis estadístico para comparar los vientos entre todas las estaciones se realizó en función de la frecuencia y dirección predominante, mientras que en las estaciones con observaciones simultáneas de viento y corrientes (Chiguao, Auchemó, Guabún e isla Italia). Se cuantificó la influencia del viento en la corriente residual utilizando correlaciones cruzadas (Pizarro et al. 1994).

Por otro lado, el oleaje fue analizado desde el punto de vista de series de tiempo comparando las alturas significativas (Hs) y la distribución de energía en diferentes frecuencias en las diferentes localidades siguiendo lo descrito por Bendat \& Piersol (1972). El análisis espectral integrado se realizó dividiendo las series de tiempo en trozos de igual longitud a los cuales se les extrajo la tendencia lineal y se les calculó el poder espectral. Este espectro integrado de las series de tiempo fue complementado con un análisis direccional de las alturas de olas, el que consiste en determinar la forma en que se distribuyen las alturas significativas sobre las direcciones de propagación (Komen et al. 1996, SilvaCasarín 2005).

\section{Resultados}

\section{DistribuCión de FRECUENCIAS DEL VIENTO}

\section{Seno de Reloncaví}

Los resultados de las observaciones mostraron que el régimen general de viento ( $>2$ años de datos) en la zona 
del seno de Reloncaví (estación de Metri y observaciones históricas en Puerto Montt) mostraron el predominio del viento norte $(>36 \%)$ seguido en menor proporción $(<$ $20 \%)$ por eventos de viento provenientes desde el noroeste y noreste abarcando los primeros dos cuadrantes, y una baja cantidad de eventos suroeste y $\operatorname{sur}(\sim 10 \%)$ (Fig. 2a, b). El análisis estacional evidenció que el predominio del viento desde el norte ocurre durante el invierno mientras que desde el sur-suroeste durante el verano austral. En esta zona, sobre el $40 \%$ de las magnitudes se concentraron entre 1 a $3 \mathrm{~m} \mathrm{~s}^{-1}$, mientras que en el rango entre 3 y $6 \mathrm{~m} \mathrm{~s}^{-1}$ presentaron porcentajes de entre 34,6 y 25\%, respectivamente (Fig. 3a). Entre 6 y 9 $\mathrm{m} \mathrm{s}^{-1}$, las observaciones se concentran entre el $4 \%$ y $10 \%$. Por último, observaciones con vientos mayores a $9 \mathrm{~m} \mathrm{~s}^{-1}$ solamente representaron un porcentaje menor al $5 \%$. El evento máximo registrado en la costa norte del seno de Reloncaví ocurrió durante el invierno de 2009, alcanzando un viento desde el norte de 19,9 $\mathrm{m} \mathrm{s}^{-1}$.

\section{Golfo Corcovado}

En el sector de Quellón (estación de punta Chiguao y datos históricos de isla Laytec), los histogramas representan observaciones obtenidas durante dos periodos del año, invierno (Fig. 2c y 3d) y primavera (Fig. $2 \mathrm{~d})$. Durante el invierno, predominó el viento $\left(<10 \mathrm{~m} \mathrm{~s}^{-1}\right)$ proveniente desde el cuadrante oeste, noroeste y norte (>60\%), con episodios de varios días interrumpidos por pulsos de viento sur-suroeste, los cuales aparecen cada 3 días en el registro.

En primavera permanecen las direcciones predominantes, pero la frecuencia disminuye $(<24 \%)$ mientras que la intensidad de los eventos se incrementó $\left(>20 \mathrm{~m} \mathrm{~s}^{-1}\right)$, tal que menos de un $4 \%$ de los registros se observaron eventos entre 25 y $30 \mathrm{~m} \mathrm{~s}^{-1}$, y solamente un evento extremos de $40 \mathrm{~m} \mathrm{~s}^{-1}$. Más de 30 observaciones mayores a $30 \mathrm{~m} \mathrm{~s}^{-1}$, durante primavera confirman la validez del evento extremo. Durante este periodo, el viento incidente desde el noroeste cambia de dirección hacia la componente suroeste en periodos de $\sim 10$ días.

En la costa este del golfo Corcovado (Auchemó), durante primavera-verano predominó el viento proveniente del oeste (>36\%) con influencia de eventos de viento desde el suroeste y noreste con magnitudes entre 1 y $10 \mathrm{~m} \mathrm{~s}^{-1}$ (Fig. 2h y $3 \mathrm{~g}$ ) que llegaron hasta $15 \mathrm{~m} \mathrm{~s}^{-1}$. Se observaron también eventos de viento provenientes de la región continental (este), soplando hacia el oeste-suroeste con intensidades típicamente menores a los $5 \mathrm{~m} \mathrm{~s}^{-1}$.

\section{Ancud y costa oeste de Chiloé}

En el sector de Ancud (estación de punta Guabún y datos históricos del faro Corona), los resultados de las observaciones mostraron un predominio del viento proveniente del sur (primavera) (Fig. 2e y $3 \mathrm{j}$ ) y suroeste (primavera-verano). Además, destacan relevantes eventos intensos de viento norte de corta duración, preferentemente durante los periodos invernales. El registro de faro Corona (Fig. 2f), el más extenso de todas las estaciones, mostró que las componentes sur, suroeste y este agrupan mas del $45 \%$ de los registros, mientras que los eventos de viento norte-noreste-noroeste agrupan un $42 \%$. Las magnitudes en esta localidad fueron dominadas por el rango entre 1 y $10 \mathrm{~m} \mathrm{~s}^{-1}(80 \%$ de las observaciones), seguida por un $15 \%$ de eventos entre 10 y $20 \mathrm{~m} \mathrm{~s}^{-1}, 1,3 \%$ de entre 20 y $30 \mathrm{~m} \mathrm{~s}^{-1}$, y un $0,04 \%$ de eventos mayores a $30 \mathrm{~m} \mathrm{~s}^{-1}$.

\section{Isla Italia (Canal Darwin)}

La isla Italia, ubicada en el sector occidental de Aysén (estación de isla Italia) e interior del canal Darwin fue monitoreada durante la primavera de 2009 (Fig. 2g). Durante la primavera, la observación cercana a la isla estuvo dominada por el viento proveniente desde el oeste $(51,2 \%)$, y, en menor porcentaje, por el viento desde el noroeste $(11 \%)$ y suroeste $(7,7 \%)$. En verano, predominó el viento proveniente del cuadrante suroeste $(27,5 \%)$, oeste $(28,1 \%)$ y noroeste $(23 \%)$. Las ocurrencias de magnitudes se concentraron en el rango $3-9 \mathrm{~m} \mathrm{~s}^{-1}(56,6 \%)$, en 3-6 $\mathrm{m} \mathrm{s}^{-1}(28,9 \%)$ y en $6-9 \mathrm{~m} \mathrm{~s}^{-1}(28,9 \%)$ (Fig. $2 \mathrm{~g}$ y $\left.3 \mathrm{~m}\right)$. La mayor magnitud registrada fue de $17,3 \mathrm{~m} \mathrm{~s}^{-1}$ en primavera y $18,5 \mathrm{~m} \mathrm{~s}^{-1}$ en verano, ambas correspondientes a viento desde el oeste.

Espectros de energía de la magnitud del viento presentan el dominio de varias frecuencias. En el seno de Reloncaví, se presentaron picos significativos en la banda de las 50, 25, 10, 8-7 y 4-3, 5 h (Fig. 5). En el sector de Quellón, las estaciones de punta Guabún e isla Laytec mostraron picos de energía asociados a periodos alrededor de los 50, 12 y 5,5 h (Fig. 5).

\section{DisTRIBUCIONES DIRECCIONALES DEL VIENTO Y OLEAJE}

En el seno de Reloncaví (estación de punta Metri) las alturas significativas registradas fueron relativamente pequeñas $(<0,5 \mathrm{~m})$ (Fig. $3 b)$, tomando en cuenta que solamente se logró medir por 7 días. En este mismo rango de mediciones, el oleaje de mayor altura se registró en dirección hacia el norte, generado por viento del orden 


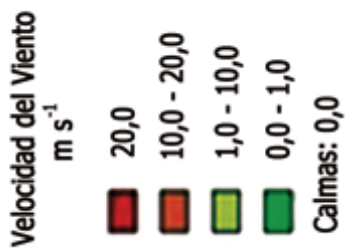
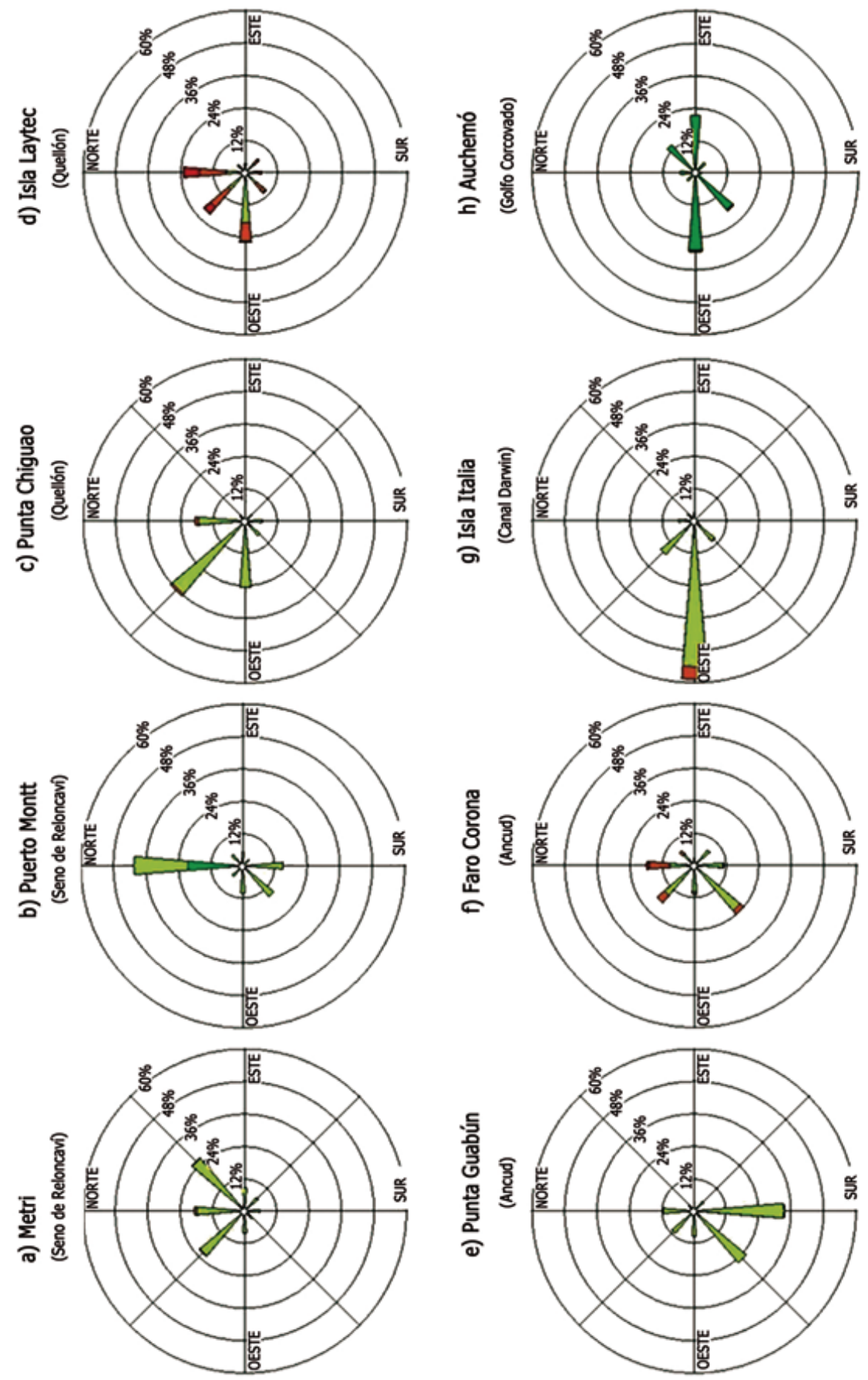

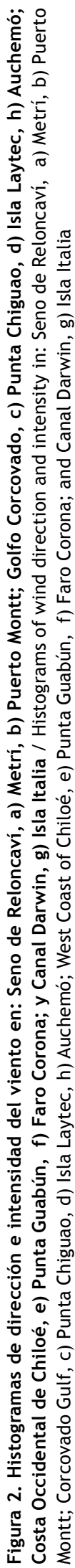




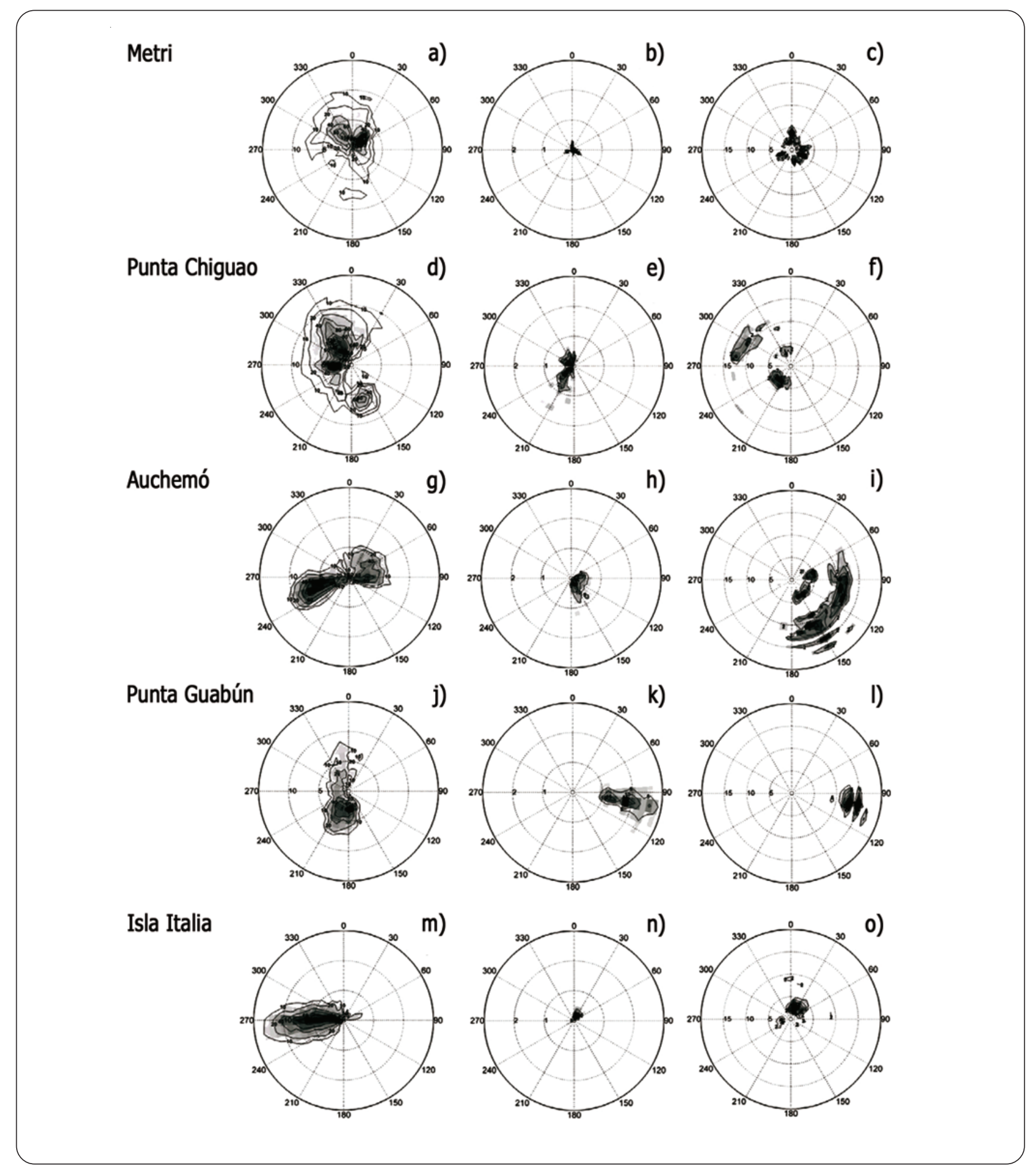

Figura 3. Distribución conjunta de viento y oleaje en las estaciones de los sectores del seno de Reloncaví (Metri), el golfo Corcovado (Punta Chiguao y Auchemó), costa occidental de Chiloé (Punta Guabún) e Isla Italia. La columna de la izquierda indica viento (magnitud en $\mathrm{m} \mathrm{s}^{-1}$ ), la del centro indica altura significativa del oleaje $(\mathrm{m})$ y la de la derecha indica período correspondiente al máximo oleaje (s) / Winds and waves in Seno de Reloncaví (Metri), Corcovado Gulf (Punta Chiguao and Auchemó), West Coast of Chiloé (Punta Guabún) and Italia Island. The left column indicates the wind magnitude $\left(\mathrm{m} \mathrm{s}^{-1}\right)$, the central column shows the significant wave height $(\mathrm{m})$ and the right column shows the maximum wave period $(\mathrm{s})$ 
de $10 \mathrm{~m} \mathrm{~s}^{-1}$ (Fig. 3a, b y Fig. 5a, comparación zonas), aunque la distribución direccional mostró que la mayor parte de los registros de oleaje no tienen dirección predominante y corresponden a periodos menores a $5 \mathrm{~s}$ (Fig. 3c). La mayor parte de la variabilidad (90\%) direccional en los valores de períodos y alturas observados en las Figuras $3 b$ y $3 c$ están asociados a errores en la estimación del espectro debido a los bajos niveles de energía observados durante el corto período de mediciones.

El sector occidental del golfo Corcovado (estación de punta Chiguao-Quellón) es una zona relativamente protegida del oleaje proveniente del Océano Pacífico. Los valores de altura significativa observada son típicamente menores a $1 \mathrm{~m}$ (Fig. 3e), con direcciones de propagación hacia el noroeste (hacia la costa) desde $120^{\circ}$ y $150^{\circ}$, coherentes con el viento proveniente de esa dirección. Este oleaje mostró periodos de máxima energía espectral entre 10 y $15 \mathrm{~s}$, es decir oleaje largo (Fig. 3f) ya que debido a la dimensiones de la zona, este oleaje largo es probablemente generado en la región oceánica el cual podría estar penetrando por la boca del Guafo (Fig. 1). Otra parte del oleaje se propaga hacia el suroeste, desde $30^{\circ}$ y $60^{\circ}$ (Fig. 3f), con períodos en torno a los $5 \mathrm{~s}$ (Fig. 3f) por lo que estaría generado localmente por una componente del viento que predomina hacia el sur (Fig. 3d).

En el sector oriental del golfo Corcovado (estación de punta Auchemó), el valor medio de altura significativa fue de $0,5 \mathrm{~m}$ (Fig. $3 \mathrm{~g}$ ) que proviene de la dirección oestenoroeste hacia el cuadrante este-sureste (Fig. 3h, i), lo que es coherente con la dirección en la que se observan las mayores intensidades de viento (Fig. 3g). De hecho, es desde el noroeste de donde se observaron las mayores alturas significativa registradas durante el periodo $(\sim 1,5 \mathrm{~m})$. El gráfico de distribución conjunta de períodos (Fig. 3i) indica al menos dos señales claras de oleaje: una señal con períodos entre 10 y $15 \mathrm{~s}$ que se propagan hacia la costa desde el cuadrante noroeste y que posiblemente este asociado al oleaje que penetra desde la región oceánica a través de la boca del Guafo y otra es una señal con período de $5 \mathrm{~s}$ que se propaga a lo largo de la costa oriental del Golfo de Corcovado.

La costa occidental de Chiloé (punta Guabún) está caracterizada por ser una zona expuesta directamente al oleaje proveniente del Océano Pacífico (Fig. 1). En esta localidad el oleaje predominante proviene del oeste, es decir de mar abierto, con mayor ocurrencia de alturas entre 1 y 2,5 m (Fig. 3k). De acuerdo a las observaciones, en algunos casos la altura significante del oleaje sobrepasa los $3 \mathrm{~m}$ con períodos de máxima energía espectral correspondiente a oleaje largo (swell) que se propaga hacia la costa. Este tipo de oleaje largo mostró periodos de 12,13 y $15 \mathrm{~s}$, con algunos eventos en el que alcanza períodos de $17 \mathrm{~s}$ (Fig. 31). Cabe señalar que por los periodos asociados al oleaje y que tiene una dirección perpendicular al viento observado durante las mediciones (Fig. 3j), este oleaje no es generado por viento local.

La zona más austral (estación isla Italia), está ubicada en una zona interior del canal Darwin, orientado en la dirección suroeste-noreste. Durante el periodo de medición, la dirección predominante del viento fue hacia el este con una distribución promedio entre 0 y $10 \mathrm{~m} \mathrm{~s}^{-1}$ (Fig. $3 \mathrm{~m}$ ), con algunos eventos de viento entre 10 y 15 $\mathrm{m} \mathrm{s}^{-1}$. A pesar de la intensidad del viento observado, el oleaje predominante solamente mostró alturas del orden de $0,6 \mathrm{~m}$ (Fig. 3n). La dirección del oleaje predominante fue hacia el noreste $\left(30^{\circ}\right)$, alineado con el canal adyacente a isla Italia (Fig. 3e). Los períodos asociados al oleaje en esta zona fueron menores a $5 \mathrm{~s}$ (Fig. 3f), es decir, producido localmente.

\section{DISTRIBUCIÓN TEMPORAL DE VIENTOS Y CORRIENTES}

Las figuras 4a, b, c y d muestran los diagramas de trazos de los vectores velocidad del viento (contorno negro), corriente total $\left(\mathrm{V}_{\mathrm{T}}\right)$ en contornos celestes y distribución de velocidades residuales $\left(\mathrm{v}_{\mathrm{r}}\right)$ para las profundidades de $1 \mathrm{~m}$ (contorno rojo), $5 \mathrm{~m}$ (contorno amarillo) y $8 \mathrm{~m}$ (contorno azul). Las velocidades fueron rotadas en la dirección preferencial asociadas al eje de máxima varianza. La corriente residual corresponde a la corriente total menos el efecto de la marea.

\section{Golfo Corcovado}

En la zona occidental del golfo (sector de Quellón), las corrientes directas obtenidas del ADCP fueron rotadas con respecto a su eje de máxima varianza $\left(\sim 11^{\circ}\right)$ el cual coincidió en todas las profundidades con la dirección que sigue la línea de la costa (Fig. 4a). La región que representa la medición de punta Chiguao muestra un dominio barotrópico de la marea en el sector costero, el cual predominó durante casi todo el periodo mostrando inversiones en la dirección de la corriente paralela al eje de máxima varianza $\left(\mathrm{V}_{\mathrm{T}}\right)$ dos veces al día con pulsos máximos de velocidades sobre los $80 \mathrm{~cm} \mathrm{~s}^{-1}$ (19 de agosto y el 1 de septiembre 2009). 
Figura 4. Los diagramas de trazos de los vectores velocidad del viento (contorno negro), corriente total ( $\mathrm{V}_{\mathrm{T}}$ : línea celeste) y distribución de velocidades residuales $\left(v_{r}\right)$ en dirección del eje de máxima varianza. La corriente residual corresponde a la corriente total menos el efecto de la marea. La mediciones se realizaron en: a) Punta Chiguao, b) Auchemó, c) Punta Guabún y d) Isla Italia / Wind vector diagrams (black contour), total current ( $\mathrm{V}_{\mathrm{T}}$ ligth blue line) and residual velocities distribution $\left(v_{r}\right)$ in the direction of the maximun variance axis. The residual current corresponds to the total current minus the tidal effect measurements made in: a) Punta Chiguao, b) Auchemó, c) Punta Guabún and d) Italia Island
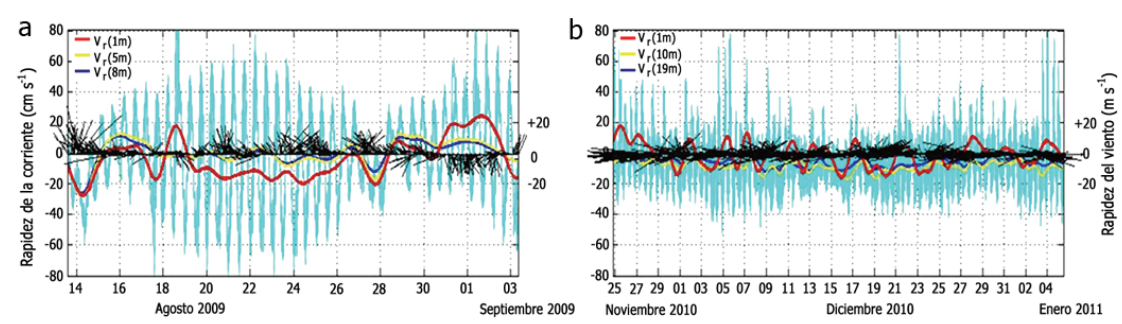

$$
\begin{aligned}
& -\mathrm{vt} \\
& - \text { viento }
\end{aligned}
$$

$C^{80}-v_{r(\text { im })}$
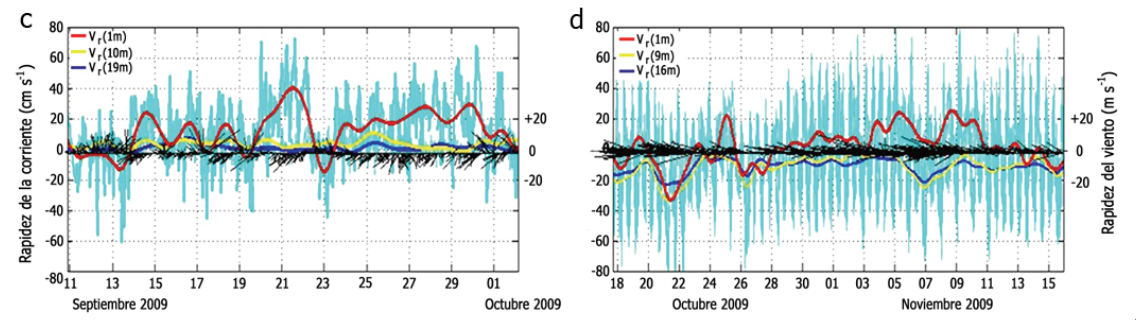

Figura 5. Series de tiempo de la altura significativa $(\mathrm{Hs})$ de las estaciones de la zona, incluye espectros de energía de la $\mathrm{Hs}$ (derecha) / Significant wave height $(\mathrm{Hs})$ time series and wave Hs energy spectrum (right side)
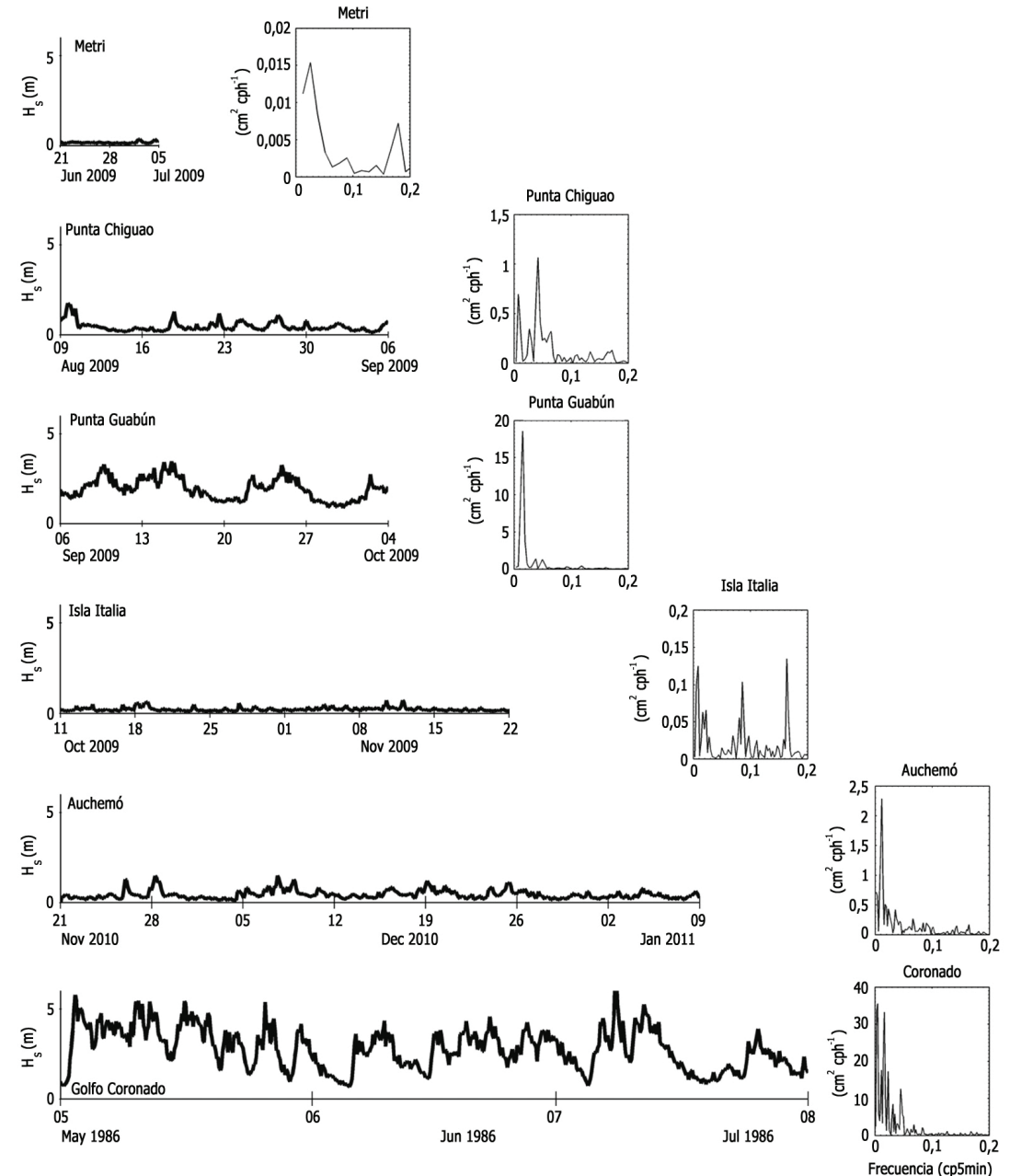
El gráfico de la corriente residual paralela al eje de máxima varianza $\left(\mathrm{v}_{\mathrm{r}}\right)$ en conjunto con el viento (diagrama de trazos) (Fig. 4a), sugiere que el viento es el principal responsable del rompimiento de la estructura que domina la marea. Durante los 3 primeros días se observó un pulso intenso de viento $\left(>18 \mathrm{~m} \mathrm{~s}^{-1}\right)$ que viene desde el $\mathrm{NW}$ el cual intensifica la corriente total y corriente residual (en toda la columna) hacia el sur, alcanzando unas magnitudes de $60 \mathrm{~cm} \mathrm{~s}^{-1}$ y $30 \mathrm{~cm} \mathrm{~s}^{-1}$, respectivamente. Este mismo evento se repite el 27-28 de septiembre. Por otro lado, durante un largo periodo (18-26 de agosto) el viento solo perturba a la corriente residual superficial $(\sim 2 \mathrm{~m})$. Finalmente, la corriente residual se desvía hacia el norte durante un evento intenso de viento SE (31 de agosto - 3 de septiembre). La alta correlación observada entre el viento meridional y la corriente residual paralela a la costa, es mostrada en la Tabla 2 y necesita solamente soplar $1 \mathrm{~h}$ para comenzar a mover la columna de agua somera.

En la figura $4 \mathrm{~b}$ se indican los campos de corrientes en la zona de Auchemó, donde las corrientes directas fueron rotadas en toda la columna con respecto a su eje de máxima varianza $\left(\sim 65^{\circ}\right)$. La región demuestra un dominio barotrópico de la marea durante todo el periodo de muestreo, donde las corrientes totales paralelas al eje de máxima varianza $\left(\mathrm{V}_{\mathrm{T}}\right)$ alcanzan un máximo $\left(>40 \mathrm{~cm} \mathrm{~s}^{-1}\right)$ durante el 3-6 de diciembre de 2009. Además, no se observan similitudes en las amplitudes con la región de Punta Chiguao, dado que en esta última las corrientes las duplican, a pesar de que ambas estaciones se encuentran ubicadas el interior del golfo Corcovado. Las mediciones de la columna de agua alcanzaron hasta los $19 \mathrm{~m}$, siendo la corriente residual paralela al eje de máxima varianza $\left(\mathrm{v}_{\mathrm{r}}\right)$ en tres profundidades menores a $10 \mathrm{~cm} \mathrm{~s}^{-1}$ y el viento menor a $10 \mathrm{~m} \mathrm{~s}^{-1}$, respectivamente.
A pesar de que las magnitudes de viento son pequeñas, son en gran parte responsable del movimiento que tiene la corriente residual superficial ( hasta los $5 \mathrm{~m}$ ), como lo muestra la alta correlación entre el viento zonal y la velocidad residual perpendicular a la costa, y entre viento meridional y la velocidad residual paralelo a la costa (Tabla 2).

\section{Sector occidental de Chiloé}

En el sector occidental de Ancud las corrientes registradas en punta Guabún tuvieron que ser rotadas con respecto a un eje de máxima varianza de $90^{\circ}$, quedando las corrientes totales $\left(\mathrm{V}_{\mathrm{T}}\right)$ y residuales $\left(\mathrm{v}_{\mathrm{r}}\right)$ orientadas perpendicular a la costa y las componentes $U_{T}$ y $u_{r}$ a lo largo de la costa. La figura $4 \mathrm{c}$ muestra las corrientes totales $\left(\mathrm{U}_{\mathrm{T}}\right)$ y residuales $\left(\mathrm{u}_{\mathrm{r}}\right)$ a lo largo de la costa. La componente a lo largo de la costa $\left(\mathrm{U}_{\mathrm{T}}\right)$ muestra una influencia significativamente menor de las mareas que las estaciones del mar interior de Chiloé. Además, se observó una directa correspondencia entre el incremento del viento y las máximas intensidades de las corrientes residuales en los primeros $6 \mathrm{~m}$ de la columna de agua. En particular se destacan dos periodos (20-22 y 24-30 de septiembre) de intensos vientos $\left(\sim 17 \mathrm{~m} \mathrm{~s}^{-1}\right)$ que alcanzan las máximas velocidades totales $\left(\mathrm{U}_{\mathrm{T}}: 70\right.$ y $\left.60 \mathrm{~cm} \mathrm{~s}^{-1}\right)$ y residuales máximas $\left(40 \mathrm{~cm} \mathrm{~s}^{-1}\right)$ durante el 22 de septiembre 2009.

La tabla 2 muestra una alta correlación entre el viento zonal $\left(\mathrm{W}_{\mathrm{x}}\right)$ y la corriente residual paralela al eje de máxima varianza $\left(\mathrm{v}_{\mathrm{r}}\right)$, similar resultado muestra el viento meridional $\left(\mathrm{W}_{\mathrm{y}}\right)$ y la corriente residual perpendicular al eje de máxima varianza $\left(\mathrm{u}_{\mathrm{r}}\right)$.

Tabla 2. Coeficientes de correlación cruzada entre viento zonal $(\mathrm{Wx})$ versus corriente residual perpendicular $\left(u_{r}\right)$ y paralela a la costa $\left(v_{r}\right)$. Coeficientes de correlación cruzada entre viento Meridional $(\mathrm{Wy})$ versus corriente residual perpendicular $\left(\mathrm{u}_{\mathrm{r}}\right)$ y paralela a la costa $\left(v_{r}\right)$ / Cross-correlation coefficients between zonal wind $(\mathrm{Wx})$ versus residual current components along $\left(v_{r}\right)$ and across the coastline $\left(u_{r}\right)$. Cross-correlation coefficients between meridional wind $(\mathrm{Wy})$ and residual current components, along $\left(\mathrm{v}_{\mathrm{r}}\right)$ and across the coastline $\left(\mathrm{u}_{\mathrm{r}}\right)$

\begin{tabular}{lcccc}
\hline Localidades & $\mathrm{C}\left(\mathrm{W}_{\mathrm{x}}, \mathrm{u}_{\mathrm{r}}\right)$ & $\mathrm{C}\left(\mathrm{W}_{\mathrm{x}}, \mathrm{v}_{\mathrm{r}}\right)$ & $\mathrm{C}\left(\mathrm{W}_{\mathrm{y}}, \mathrm{u}_{\mathrm{r}}\right)$ & $\mathrm{C}\left(\mathrm{W}_{\mathrm{y}}, \mathrm{v}_{\mathrm{r}}\right)$ \\
\hline $\begin{array}{l}\text { Chiguao } \\
\text { Guabún }\end{array}$ & +1 (lag: $-1 \mathrm{~h})$ & -1 (lag: $-1 \mathrm{~h})$ & -1 (lag: $-1 \mathrm{~h})$ \\
Isla Italia & $-1($ lag: $-0.96 \mathrm{~h})$ & & \\
Auchemó & & -1 (lag: $-1 \mathrm{~h})$ & -1 (lag: $-1 \mathrm{~h})$ \\
\hline
\end{tabular}




\section{INTERIOR DE CANAL DARWin (SECTOR ISLA ItALia)}

En el sector de isla Italia el sistema de viento y corriente presentó una alta variabilidad (Fig. 4d) con un predominio del viento proveniente del suroeste, oeste y noroeste durante casi todo el periodo de muestreo. Las corrientes superficiales totales y residuales fueron rotadas con respecto al eje de máxima varianza $\left(\sim 60^{\circ}\right)$, quedando las componentes $\left(\mathrm{V}_{\mathrm{T}} \mathrm{y} \mathrm{u}_{\mathrm{r}}\right)$ paralelas a la costa de la isla, donde se encuentra la mayor energía asociada a la marea, en cambio en la componente perpendicular, las velocidades no superan los $10 \mathrm{~cm} \mathrm{~s}^{-1}$. Nuevamente, al igual que en las otras estaciones se observaron dos forzantes (mareas y vientos) aunque, las corrientes totales $\left(\mathrm{V}_{\mathrm{T}}\right)$ son dominadas principalmente por las mareas mostrando un comportamiento completamente barotrópico y alcanzan un máximo de $70 \mathrm{~cm} \mathrm{~s}^{-1}$ especialmente evidente durante los periodos 2-3 y 11-14 de noviembre 2009 .

La corriente residual más profunda, bajo los $3 \mathrm{~m}$, muestra un comportamiento barotrópico durante todo el periodo de mediciones, sin embargo, la corriente superficial a lo largo del canal muestra las mayores magnitudes y en sentido opuesto con respecto a las capas de fondo. Este resultado, está asociado al viento, debido a la alta correlación opuesta (-1) entre la corriente residual a lo largo de la costa $\left(\mathrm{u}_{\mathrm{r}}\right)$ y el viento zonal $\left(\mathrm{W}_{\mathrm{x}}\right)$.

En general, en las estaciones ubicadas en las zonas interiores, la marea explica la mayor parte de la variabilidad de las corrientes, tal como ocurre en Chiguao con el 76\% y en Auchemó el 55\% aunque en isla Italia la marea explica el 46\%. La estación de Guabún, que se encuentra expuesta a la influencia del Océano Pacífico la marea solamente explica el $42 \%$ de la variabilidad de las corrientes. Por otro lado, las corrientes residuales son explicadas íntegramente por el viento ya que corresponden a estaciones costeras y someras $(<40 \mathrm{~m})$ donde el gradiente de presión no es relevante en relación al esfuerzo que ejerce el viento.

\section{Discusión}

La zona en estudio muestra una alta complejidad topográfica, desde el punto de vista de la batimetría y de las elevaciones continentales, por lo que es necesario resaltar algunas de estas características en las zonas analizadas, con el fin de entender la influencia que ejercen en los parámetros oceanográficos-atmosféricos medidos. El seno de Reloncaví es un sistema semicerrado, de forma casi-circular con un diámetro promedio de $30 \mathrm{~km}$, con aportes de agua desde el fiordo de Reloncaví y conectado hacia el sur con el golfo de Ancud a través de los pasos Queullín y Nao (Cáceres et al. 2010). La costa norte del seno de Reloncaví, donde se ubicó la estación de Metri y se obtuvo la información histórica de Puerto Montt, fue la que presentó mayores profundidades $(350 \mathrm{~m})$ asociadas a un canal que se proyecta desde la boca del fiordo hacia el noroeste, siguiendo la línea costera (Rodrigo 2006). La zona de mediciones presentó una pendiente abrupta desde la línea costera hacia el canal que llega hasta la plataforma de Puerto Montt que registra profundidades menores de $50 \mathrm{~m}$ y una pendiente suave. Hacia el centro y sur del seno de Reloncaví el fondo se eleva a profundidades menores de $50 \mathrm{~m}$, formando una plataforma con elevaciones que forman islas. Esta plataforma es interrumpidas por un canal $(\sim 100 \mathrm{~m})$ que cruza el seno de Reloncaví conectando la cara noreste del seno con el paso Queullín.

El golfo Corcovado, en el cual se registraron observaciones en la costa este (Chiguao) y oeste (Auchemó), es una porción de agua que limita al norte por las islas del archipiélago de Quinchao, al oeste por el continente y al este por la isla de Chiloé, mientras que al suroeste está conectado con el Océano Pacífico por la Boca del Guafo. Esta zona es más somera que el seno de Reloncaví con profundidades del orden de $170 \mathrm{~m}$ y amplias plataformas $(50 \mathrm{~m})$ especialmente en el sector occidental del Golfo. Sobre estas plataformas y en caras opuestas del golfo se ubicaron las estaciones de Chiguao y Auchemó. Particularmente, Chiguao está limitada topográficamente al oeste por los montes con alturas superiores a $100 \mathrm{~m}$ de la isla de Chiloé, mientras que Auchemó está limitada al este por la cordillera de los Andes (Rodrigo 2006). En el sector noroeste de la isla de Chiloé, la estación de Guabún y las zonas de registros históricos de faro Corona y golfo Coronado se ubican en la entrada del canal Chacao expuestas a la influencia del viento y olas provenientes del Pacífico e influenciadas por la orientación del canal. En forma similar, el sector de isla Italia corresponde a una cuenca de $9 \mathrm{~km}$ de extensión al interior del canal Darwin, de $20 \mathrm{~km}$ de largo, $3 \mathrm{~km}$ de ancho y $160 \mathrm{~m}$ de profundidad en orientación noroestesureste que conecta con el Pacífico. Esta cuenca de $70 \mathrm{~m}$ de profundidad tiene una pendiente abrupta desde la costa norte donde se ubica la estación de medición (carta 8000 $\mathrm{SHOA})^{3}$.

\footnotetext{
${ }^{3}$ SHOA. 2001. Carta 8000. Isla Guafo a Golfo de Penas. Servicio Hidrográfico y Oceanográfico de la Armada de Chile, Valparaíso.
} <www.shoa.cl> 
La gran extensión espacial de la zona de estudio (Fig. 1) permitió solamente percibir una parte limitada de la distribución del campo de vientos. A pesar de lo anterior, al considerar el análisis por zonas (e.g., Seno de Reloncaví y Golfo Corcovado), los registros de viento mostraron coherencia geográfica entre datos históricos y registros tomados durante el desarrollo de las campañas de medición. Esta coherencia ocurrió a pesar de que difieren los periodos de medición. Así, la cuenca del seno de Reloncaví está caracterizada por el predominio del viento proveniente del sector noroeste-norte-noreste. El lado occidental del golfo Corcovado, se caracteriza por vientos del sector norte-noroeste-oeste, mientras que en la parte oriental del golfo predominan vientos desde el oeste y suroeste provenientes de la boca del Guafo y modulados con eventos de viento desde el continente. En el lado occidental de la isla de Chiloé el viento predominante proviene desde el sur y suroeste con eventos sinópticos de viento norte, especialmente durante el invierno austral (Cáceres et al. 2003).

Las observaciones, en todas las estaciones, sugieren que la topografía de la zona tiene una fuerte influencia en el predominio de la dirección del viento local en los diferentes sectores. Los resultados mostraron que en la zona exterior de Chiloé, el viento estaría controlado por la circulación atmosférica regional asociada al movimiento latitudinal del anticiclón del Pacífico Sur y la deriva de los vientos del oeste (Chaigneau \& Pizarro 2005, Letelier et al. 2010), como también el paso eventual de sistemas frontales de viento norte (Letelier 2010). Por otro lado, en el sector de Quellón (occidente del golfo Corcovado) a sólo $100 \mathrm{~km}$ al este, la dirección predominante fue $\sim 45^{\circ}$ respecto de la zona exterior de Chiloé. Este cambio en la dirección está determinado por la presencia de los montes $(>100 \mathrm{~m})$ de la isla de Chiloé que interrumpen la influencia directa del Océano Pacífico (Garreaud \& Muñoz 2005) sobre el sector oriental del golfo Corcovado. El efecto de la topografía queda claramente de manifiesto en la estación de faro Corona (registros históricos), ya que aparecen direcciones secundarias siguiendo la orientación del canal Chacao. Otro ejemplo se observó en la zona más austral y aislada del resto de las mediciones: en isla Italia ubicada $30 \mathrm{~km}$ al interior del canal Darwin predominó del viento desde el oeste, siguiendo el eje topográfico zonal oesteeste del Canal.

Al igual que el viento, el oleaje registrado en las diferentes localidades presentó el predominio de características locales, oceánicas o mixtas (Fig. 5). En el seno de Reloncaví se observó esencialmente oleaje local (Tabla 3) generado por viento local dentro de la cuenca que tiene aproximadamente 35 a $40 \mathrm{~km}$ de diámetro. A consecuencia de lo anterior se generó oleaje de baja altura $(<0,5 \mathrm{~m})$ con periodos menores a $5 \mathrm{~s}$. Estas observaciones concuerdan con los cálculos realizados en la zona con la metodología de Massel (Massel 1996, Soto-Mardones et al. 2009).

En el golfo Corcovado se observó oleaje de característica mixta: uno de periodo menor a $5 \mathrm{~s}$ de origen local y otro de periodo mayor a $10 \mathrm{~s}(10-15 \mathrm{~s})$ el que no fue generado localmente debido a las dimensiones del golfo $(\sim 50 \mathrm{~km})$, sino que fue generado en la región oceánica y penetró por la boca del Guafo. Evidencia clara de este proceso, es que aunque las alturas significativas son similares entre la costa oeste (Quellón) y este del golfo (Auchemó), en esta última predominan periodos mayores y en la primera el oleaje dominante viene del sureste. Los procesos de generación, propagación y reflexión del oleaje dentro del golfo, fueron analizados usando un modelo numérico de olas en aguas somera (SWAN).

Tabla 3. Clasificación de estaciones por características de oleaje observado / Station ranking according to the observed wave characteristics

\begin{tabular}{lccc}
\hline Estación & Categoría & Predominio Oleaje & $\begin{array}{c}\text { Forzante de } \\
\text { Corrientes }\end{array}$ \\
\hline $\begin{array}{l}\text { Punta Metri } \\
\text { Punta Chiguao }\end{array}$ & Protegida & Local & \\
Auchemó & SemiProtegida & Mixto & Marea \\
Punta Guabún & Expuesta & Mixto & Marea \\
Golfo Coronado & Expuesta & Largo (Oceánico) & Viento \\
Isla Italia & Protegida & Local & Viento \\
\hline
\end{tabular}


En la zona occidental de Ancud (punta Guabún y golfo Coronado), costa occidente de Chiloé, las observaciones realizadas y datos históricos mostraron que el oleaje es principalmente del océano abierto tal como lo describieron Cabezas (1991) y Belmar (2002). Las observaciones mostraron olas con alturas significativas que sobrepasan los $2 \mathrm{~m}$ e incluso eventualmente los $3 \mathrm{~m}$ con períodos de entre 12 y $17 \mathrm{~s}$, los que corresponden a oleaje largo (Swell) que se propaga hacia la costa. En esta zona registros históricos confirman la altura del oleaje típico encontrado, pero también evidencian eventos de oleaje en los que se han observado alturas mayores a $6 \mathrm{~m}$ en presencia de un sistema frontal extremo (Cabezas 1991). La altura del oleaje observada en esta zona es consistente con el incremento de altura hacia el sur de la costa de Chile (Belmar 2002). El principal origen del oleaje de largo periodo en esta zona son los sistemas frontales que se mueven hacia el este asociados al límite sur del anticiclón del Pacífico Sur (la deriva de los vientos del oeste) (Estienne \& Godard 1970, Jara et al. 1994). El decaimiento de la altura del oleaje hacia el norte obedece a que se encuentra a mayor distancia de la zona de generación y por lo tanto se incrementa la importancia del oleaje generado localmente.

En la zona más austral (isla Italia), el régimen de oleaje observado sugiere la presencia de una zona protegida, igual que el seno de Reloncaví, la que está fuertemente influenciada por la topografía (especialmente el canal Darwin) y el oleaje formado localmente (Hs $<0,60 \mathrm{~m}$ ). La altura y período del oleaje largo proveniente del Océano Pacífico podría ser atenuado por la topografía, es decir, por el efecto combinado de la fricción de fondo, rompimiento de ola, refracción y el bloqueo de olas por la línea costera, como lo demuestran mediante modelación numérica y observaciones Caliskan \& Valle-Levinson (2008).

Al igual que en los otros parámetros, la topografía parece jugar un rol preponderante en la dirección del campo de corrientes costeras, ya que en la zona costera del golfo Corcovado e isla Italia, el eje de máxima varianza de las corrientes no coincidió con los ejes geográficos, sino que con la dirección paralela y perpendicular a la costa. Los resultados mostraron que en estas zonas (Seno de Reloncaví y el Golfo Corcovado) el principal forzante de la corriente es la marea, ya que representan más del $76 \%$ y $55 \%$ de su variabilidad. Esto ocurre a diferencia de la zona exterior de Chiloé donde la marea pierde importancia en la conducción de las corrientes costeras (42\%). Cabe señalar que en la zona del canal Darwin (isla Italia), aunque predomina el oleaje local, la marea solamente explica el $46 \%$ de la variabilidad de las corrientes debido a la intensidad del viento oeste. A pesar de la intensidad del viento registrado, el oleaje largo no se puede desarrollar debido a la limitada extensión de la zona (Soto-Mardones et al. 2009), pero si tiene la capacidad de conducir las corrientes costeras. Esta conclusión en base a observaciones, es consistente con los resultados previos de un modelo numérico regional que mostró que la variabilidad espacial de la batimetría del mar interior de la región norte de los fiordos Patagónicos $\left(41^{\circ}-46^{\circ} \mathrm{S}\right)$ es un factor determinante en la diferencia de amplitud que alcanza la marea entre las diferentes cuencas lo que podría modular la intensidad de las corrientes en la zona (Aiken 2008). Por lo anterior, la variabilidad e intensidad de las corrientes costeras en la región norte de los fiordos Patagónicos de Chile están moduladas por el efecto topográfico y batimétrico a diferencia de la zona expuesta de Chiloé donde el principal forzante es el viento.

En términos energéticos los resultados muestran que las corrientes dominan en la zona interior de la región norte de los fiordos Patagónicos, mientras que en el sector occidental de la isla de Chiloé la mayor fuente de energía sobre la plataforma continental es el oleaje largo generado remotamente. Dada la complejidad y extensión de la zona, las observaciones analizadas son un primer avance, usando mediciones directas, para entender la dinámica general del campo de viento, las corrientes y olas en la zona. El enfoque en términos de resolver aspectos generales de la zona necesariamente implica una etapa de mediciones de mayor cobertura espacial y temporal en conjunto con la implementación y validación de modelos atmosféricos y oceánicos.

\section{Agradecimientos}

Agradecemos al Dr. Felipe Hurtado (Pontificia Universidad Católica de Valparaíso) por la confianza y el apoyo en las operaciones de nuestro grupo para la toma de información en terreno. Al Dr. Alfredo Klempau (Universidad de Concepción) por sus gestiones para la realización del proyecto. Al Dr. Hugo Arancibia (Universidad de Concepción) por incorporarnos como el grupo oceanografía desde la primera fase del proyecto. Queremos agradecer al proyecto Consorcio Tecnológico de Acuicultura en Zonas Expuestas (CTUE-06-03) financiado por Conicyt y a la empresa EcoSea Farming S.A. continuadora del proyecto por la confianza, el apoyo financiero, técnico y operacional para la toma de información en terreno y desarrollo de artículos científicos. 


\section{LITERATURA CITADA}

Aiken CM. 2008. Barotropic tides of the Chilean Inland Sea and their sensitivity to basin geometry. Journal of Geophysical Research 113 (C08024): <doi:10.1029/ 2007JC004593>

Bakun A \& CS Nelson. 1991. The seasonal cycle of windstress curl in subtropical eastern boundary current regions. Journal of Physical Oceanography 21(12): 1815-1834.

Beier E. 1997. A numerical investigation of the annual variability in the Gulf of California. Journal of Physical Oceanography 27: 615-632.

Beier E \& P Ripa. 1998. Seasonal gyres in the northern Gulf of California. Journal of Physical Oceanography 29: 305-311.

Belmar JP. 2002. Distribución de parámetros espectrales de olas generadas por el viento frente a la costa de Chile (18 $\left.36^{\circ} \mathrm{S}\right)$. Tesis de Oceanógrafo, Universidad Católica de Valparaíso, Valparaíso, 98 pp.

Bendat JS \& AG Piersol. 1972. Random data: Analysis and measurement procedures, $407 \mathrm{pp}$. Wiley-Interscience, New York.

Berón-Vera FJ \& P Ripa. 2000. Three-dimensional aspects of the seasonal heat balance in the Gulf of California Journal of Geophysical Research 105: 441-457.

Berón-Vera FJ \& P Ripa. 2002. Seasonal salinity balance in the Gulf of California. Journal of Geophysical Research 107: $1-15$

Cabezas A. 1991. Características y variabilidad del régimen de oleaje en el golfo Coronados, $\mathrm{X}^{\mathrm{a}}$ región. Tesis de Oceanógrafo, Pontificia Universidad Católica de Valparaíso, Valparaíso, $106 \mathrm{pp}$.

Cáceres M, A Valle-Levison \& L Atkinson. 2003. Observations of cross-channel structure of flow in an energetic tidal channel. Journal of Geophysical Research 108(C4): 3114. <doi:10.1029/2001JC000968 >

Cáceres M, A Valle-Levinson, JP Belmar, M Bello \& M Castillo. 2010. Variabilidad transversal del flujo y salinidad en paso Nao. Ciencia y Tecnología del Mar 33(2): 45-58.

Caliskan H \& A Valle-Levinson. 2008. Wind-wave transformation in an elongated bay. Continental Shelf Research 28: 1702-1710.

Castro R. 2001. Variabilidad termohalina e intercambios de calor, sal y agua en la entrada al Golfo de California. Tesis de Doctor, Universidad Autónoma de Baja California, Ensenada, México, 212 pp.

Castro R, M Lavin \& P Pipa. 1994. Seasonal heat-balance in the Gulf of California. Journal of Geophysical Research 99(C2): 3249-3261.
Castro LR, MA Cáceres, N Silva, MI Muñoz, R León, M Landaeta \& S Soto-Mendoza. 2011. Short-term variations in mesozooplankton, ichthyoplankton, and nutrients associated with semi-diurnal tides in a patagonian Gulf. Continental Shelf Research 31: 282-292.

Chaigneau A \& O Pizarro. 2005. Surface circulation and fronts of the South Pacific Ocean, east of $120^{\circ} \mathrm{W}$. Geophysical Research Letters 32: L08605, <doi:10.1029/ 2004GL022070>

Estienne P \& A Godard. 1970. Climatologie, 368 pp. Armand Colin, París.

Foreman M. 1977. Manual for tidal heights analysis and prediction. Pacific Marine Science Report 77-10: 1-66. Institute of Ocean Sciences, Patricia Bay, Sydney.

Foreman M. 1978. Manual for tidal currents analysis and prediction. Pacific Marine Science Report 78-6: 1-57. Institute of Ocean Sciences, Patricia Bay, Sydney.

Garreaud RD \& R Muñoz. 2005. The low-level jet off the west coast of subtropial South America: structure and variability. Monthly Weather Review 133: 2246-2261.

Godin G. 1972. The analysis of tides, 264 pp. University of Toronto Press, Toronto.

González MJ \& M Cáceres. 2009. Bifurcación de la pluma estuarina del fiordo Aysén en su trayectoria sobre el canal Moraleda. Ciencia y Tecnología del Mar 32(1): 5-25.

Hasselmann K, TP Barnett, E Bouws, H Carlson, DE Cartwright, K Enke, JA Ewing, H Gienapp, DE Hasselmann, P Kruseman, A Meerburg, P Müller, DJ Olbers, K Richter, W Sell \& H Walden. 1973. Measurements of windwave growth and swell decay during the Joint North Sea wave project (JONSWAP), 93 pp. Repository Hydraulic Engineering Reports, Deutches Hydrographisches Institut, Hamburg.

Jara X, R Aldunate, H Vergara, M Cáceres, J Fierro \& $\mathbf{P}$ Bilbao. 1994. Atlas oceanográfico para la educación, 129 pp. Servicio Hidrográfico y Oceanográfico de la Armada, Valparaíso.

Komen GJ, L Cavaleri, M Donelan, K Hasselmann, S Hasselmann \& PAEM Janssen. 1996. Dynamics and modelling of ocean waves, $555 \mathrm{pp}$. Cambridge University Press, New York.

Letelier J. 2010. Surgencia y estructuras de mesoescala frente a Chile $\left(18^{\circ}-42^{\circ} \mathrm{S}\right)$ Tesis de Doctor en Oceanografía, Universidad de Concepción, Concepción, 83 pp.

Letelier J, S Hormazábal, S Núñez \& O Pizarro. 2010. Marco oceanográfico. En: Serra R (ed) Estudio de migración del jurel, Fase I, 2007. Informe Final FIP 2007-27: 1-461. 
Leth O. 2000. Ocean circulation in the Eastern South Pacific: An observational and numerical modelling study. Tesis Ph.D., University of Copenhagen, Copenhagen, 300 pp.

Massel S. 1996. On the largest wave height in water of constant depth. Ocean Engineering 23(7): 553-573.

Pawlowicz R, B Beardsley \& S Lentz. 2002. Classical tidal harmonic analysis including error estimates in MATLAB using T-TIDE. Computers \& Geosciences 28: 929-937.

Pizarro O, S Hormazábal, A González \& E Yáñez. 1994. Variabilidad del viento, nivel del mar y temperatura en la costa norte de Chile. Investigaciones Marinas 22: 85-101.

Rodrigo C. 2006. Topografía submarina en canales de la Patagonia norte. En: N Silva \& S Palma (eds). Avances en el conocimiento oceanográfico de las aguas interiores chilenas, Puerto Montt a Cabo de Hornos, pp. 19-23. Comité Oceanográfico Nacional, Pontificia Universidad Católica de Valparaíso, Valparaíso.

Salinas S \& S Hormazábal. 2003. Capacidad de transporte de la constricción de Meninea para un flujo de dos capas y el efecto de la corriente de marea. Ciencia y Tecnología del Mar 27(1): 5-15.

Silva-Casarín R. 2005. Análisis y descripción estadística del oleaje, 186 pp. Serie Docencia, Universidad Nacional Autónoma de México, Ciudad de México.
Silva N \& S Palma. 2006a. Avances en el conocimiento oceanográficos de las aguas interiores chilenas, Puerto Montt a cabo de Hornos, 162 pp. Comité Oceanográfico Nacional, Pontificia Universidad Católica de Valparaíso, Valparaíso.

Silva N \& S Palma. 2006b. El programa CIMAR en los canales y fiordos australes. En: Silva N \& S Palma (eds). Avances en el conocimiento oceanográfico de las aguas interiores chilenas, Puerto Montt a cabo de Hornos, pp. 11-15. Comité Oceanográfico Nacional, Pontificia Universidad Católica de Valparaíso, Valparaíso.

Soto-Mardones L, SG Marinone \& A Pares-Sierra. 1999. Variabilidad espacio temporal de la temperatura superficial del mar en el golfo de California. Ciencias Marinas 25(1): 1-30.

Soto-Mardones L, J Letelier, S Salinas, E Pinillas \& J Belmar. 2009. Análisis de parámetros oceanográficos y atmosféricos del Seno de Reloncaví. Gayana 73(1): 141-155.

Strub P, J Mesías, V Montecino, J Rutlland \& S Salinas. 1998. Coastal ocean circulation off western South America. En: Robinson AR \& KH Brink (eds). The sea: The global coastal ocean, regional studies and synthesis, pp. 273-313. John Wiley and Sons, New York.

Valle-Levinson A, N Sakar, R Sanay, D Soto \& J León. 2007. Spatial structure of hydrography and flow in a Chilean fjord, Estuario Reloncavi. Estuaries and Coasts 30(1): 113-126.

Recibido el 24 de marzo de 2011 y aceptado el 17 de octubre de 2011 